\title{
AN AMPEROMETRIC TECHNIQUE FOR RECORDING ASCORBATE DILUTION CURVES AND BLOOD FLOW PULSES
}

BY

\author{
P. G. F. NIXON, G. A. HAY, F. HEPBURN, H. M. SNOW, AND R. ADDYMAN \\ From the Departments of Thoracic Surgery and Medical Physics in the General Infirmary at Leeds
}

Received May 25, 1962

In 1960 Leland Clark reported his discovery that the concentration of ascorhic acid injected into the blood stream could be measured continuously with a platinum electrode and amperometric apparatus. His communication was important because it gave cardiologists an innocuous indicator and a simple method for recording dilution curves within the circulation. The principle of the method is that a constant potential difference is maintained between a platinum electrode introduced into the blood stream and a reference electrode placed on the skin. When injected vitamin C reaches the platinum electrode some ascorbate ions are oxidized, additional electrons are released, and the current increases proportionally to the concentration of ascorbate ions at the surface of the electrode (Frommer, Pfaff, and Braunwald, 1961). The apparatus developed here for maintaining constant voltage allows the changes in current to be recorded simply and inexpensively with the later D.C. stages of commercial electrocardiographs (Hay, Hepburn, and Nixon, 1963). The apparatus also gives an estimate of the pattern of pulsatile blood flow (Pfaff, Frommer, and Morrow, 1960), and this may be recorded simultaneously with the pressure pulse at cardiac catheterization. The purpose of this communication is to report experience with the ascorbate dilution technique, and to suggest that the blood flow pulse is of value in the investigation of patients with heart disease.

\section{Material AND Methods}

The Control Unit. The potential difference between the intravascular and skin electrodes is maintained at about 1 volt, with fluctuation during any recording maintained at less than $10 \mathrm{mV}$, by a unit (Fig. 1) containing a battery and a transistor "emitter follower" stabilizing circuit. The stabilizing circuit has been designed to eliminate the need for highly sensitive recorders, and recordings may be made with the later stages of electrocardiographs requiring as much as $250 \mathrm{mV}$ for full-scale deflection. The risk of ventricular fibrillation induced by the electrode voltage is reported to be limited because the electrode current is prevented from exceeding 0.6 mA (Frommer et al., 1961). The unit has gain and baseline shift controls, and provision for the electrode region to be primed with a voltage of approximately $1.3 \mathrm{~V}$ to accelerate baseline stability.

The Electrodes. The intravascular platinum electrode may be embedded in a cardiac catheter or inserted through an indwelling needle into a peripheral artery (Pfaff et al., 1960). A catheter electrode was improvised from a double-lumen cardiac catheter (Nixon and Snow, 1960), but now single-lumen catheters are manufactured with a platinum ring embedded $2-3 \mathrm{~mm}$. behind the tip, in sizes ranging from $5 \mathrm{~F}$ to $9 \mathrm{~F}$, by the United States Instrument and Catheter Corporation. Our electrode made for insertion through a Cournand needle consists at present of a length of 28 gauge platinum wire cemented into nylon tubing. Messrs. Shrimpton and Fletcher of Redditch have agreed to manufacture an improved instrument. A standard electrocardiograph plate is used as a skin-reference electrode. 


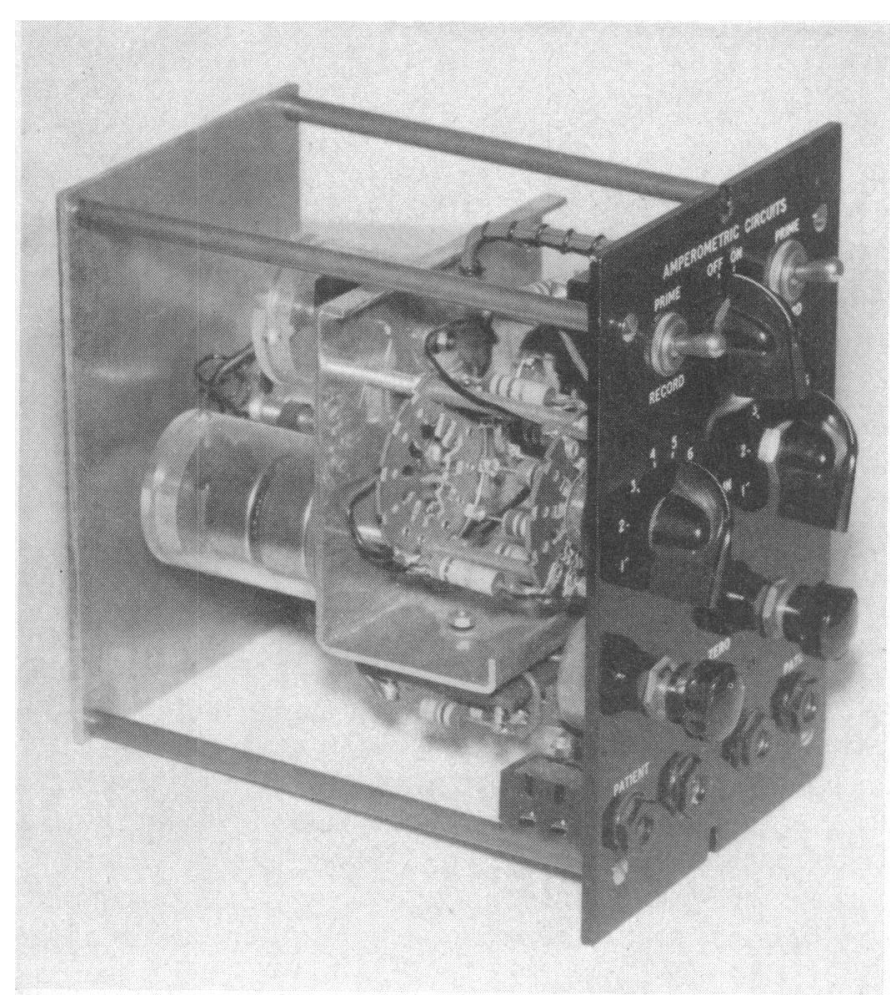

FIG. 1.-A two-channel control unit.

Operation. While one electrode may be placed inside the heart, it is advisable to ensure that no risk arises from the use of the unit in conjunction with other electrical equipment. For instance, current to earth sufficient to stimulate the heart may pass via the internal electrode when certain types of electrocardiograph are in use.

The reference electrode is strapped to the skin and the platinum electrode is introduced into the circulation. Leads are taken from the control unit to these electrodes and to the electrocardiograph. The control unit is switched on and set to "Prime" for 2030 seconds before setting to "Record". The shift control is then used to balance out the steady signal causing displacement of the baseline. The baseline is not smooth but presents a flow pulsation, subject to distortion, which may be amplified and recorded simultaneously with the pressure pulse that is transmitted through the lumen of the catheter. Before ascorbic acid is injected the gain is adjusted to give a flow pulse amplitude of 5-10 per cent of fullscale deflection. The dose of ascorbic acid is $100-200 \mathrm{mg}$. per injection.

When the dilution curve is planned to demonstrate the chamber of arrival of a left-to-right shunt the electrode is situated at the tip of the cardiac catheter and the ascorbic acid is injected through that catheter into the same cardiac chamber. To distinguish between aortic regurgitation and patent ductus arteriosus in cases of ventricular septal defect the catheter tip electrode is placed in the pulmonary artery, and the ascorbic acid is injected into the aorta through an Ŏdman-Ledin catheter introduced percutaneously via the femoral artery. To record arterial dilution curves the electrode is placed in the brachial artery, and the ascorbic acid is injected into one of the great vessels or the heart.

\section{RESULTS}

Some 200 ascorbate dilution curves have been recorded without mishap at cardiac catheterization in 70 patients. As much as $2.5 \mathrm{~g}$. ascorbic acid has been injected without detectable ill effect.

The Demonstration of Chamber of Entry of Left-to-right Shunts. When the electrode is situated at the tip of the catheter, and when ascorbic acid is injected through the catheter into a chamber that does not receive shunted blood, the dilution curve has the appearance of the right atrial and inferior vena caval curves illustrated in Fig. 2. The initial deflection (I) marks the time of injection and is caused by the ascorbic acid leaving the catheter tip and washing over the electrode. The second deflection (S) is caused by ascorbic acid returning to the electrode after traversing the systemic circulation. When the site of electrode and injection is a chamber of the right heart that receives shunted blood, the curve resembles the right ventricular and pulmonary arterial curves in Fig. 2. The initial deflection (I) is followed by a pulmonary recirculation peak $(\mathrm{P})$ caused by ascorbic acid returning prematurely to the electrode, via a shunt, after traversing the pulmonary circulation. Thus in atrial septal defect the pulmonary recirculation peak occurs in the right 
atrium and ventricle and pulmonary artery; in ventricular septal defect it occurs in the right ventricle and pulmonary artery; and in aorto-pulmonary communications it is found in the pulmonary artery (Fig. 3).

The technique appears to detect very small left-to-right shunts.

Case Report. An emigrant of 18 years was referred for diagnosis of his cardiac lesion. There were no symptoms and the only abnormal sign was a murmur at the left sternal border. The murmur began with the first heart sound but did not extend to the second sound. The electrocardiogram and chest radiograph were normal. Cardiac catheterization revealed normal pressure and 72-75 per cent oxygen saturation in the caval veins, right heart, and pulmonary artery: the ear-piece indicatordilution curve obtained by injecting blue dye into the pulmonary artery was normal (Fig. 4). The entry of a left-to-right shunt into the right ventricle was demonstrated by catheter-tip ascorbate curve (Fig. 5) and by hydrogen inhalation (Clark and Bargeron, 1959).

Occasionally it is impossible to decide whether or not shunted blood enters a particular chamber because unusual prolongation of the downslope of the initial curve masks the site of the pulmonary recirculation peak. This difficulty usually disappears when the catheter-tip is moved to another part of the chamber. If changing the position of the platinum electrode and repeating the injection do not solve the problem, the question of the entry of a left-to-right shunt is decided by using the same electrode to record the appearance time of inhaled hydrogen (Clark and Bargeron, 1959). For this purpose the reference and catheter-tip electrodes are connected directly to the D.C. stage of the electrocardiograph.

Diagnosis of Aortic regurgitation and Patent Ductus Arteriosus in Ventricular Septal Defect. In ventricular septal defect an early diastolic murmur is probably caused by aortic regurgitation, patent ductus arteriosus, or pulmonary regurgitation. To determine which of these conditions is likely to be present the catheter-tip electrode is placed in the main pulmonary artery and an Odman-Ledin catheter is introduced into the aorta by the Seldinger technique. Ascorbic acid is injected into the aorta at two sites, the one just above the aortic valve, the other at the site of the ductus. When aortic regugitation is present, some of the ascorbic acid injected close to the aortic valve regurgitates into the left ventricle and passes through the ventricular septal defect to reach the electrode prematurely, many seconds before it could have reached the pulmonary artery by traversing the systemic circulation. The ascorbic acid injected at the site of the closed ductus tends to flow onwards around the systemic circulation without regurgitating sufficiently to arrive prematurely at the pulmonary artery (Fig. 8). When the ductus arteriosus is patent the ascorbic acid injected close to its origin reaches the electrode prematurely and with a concentration greater than when the injection is made close to the aortic valve (Fig. 6). When pulmonary regurgitation is the cause of the early diastolic murmur the electrode in the pulmonary artery is not reached prematurely by ascorbic acid injected into the aorta.

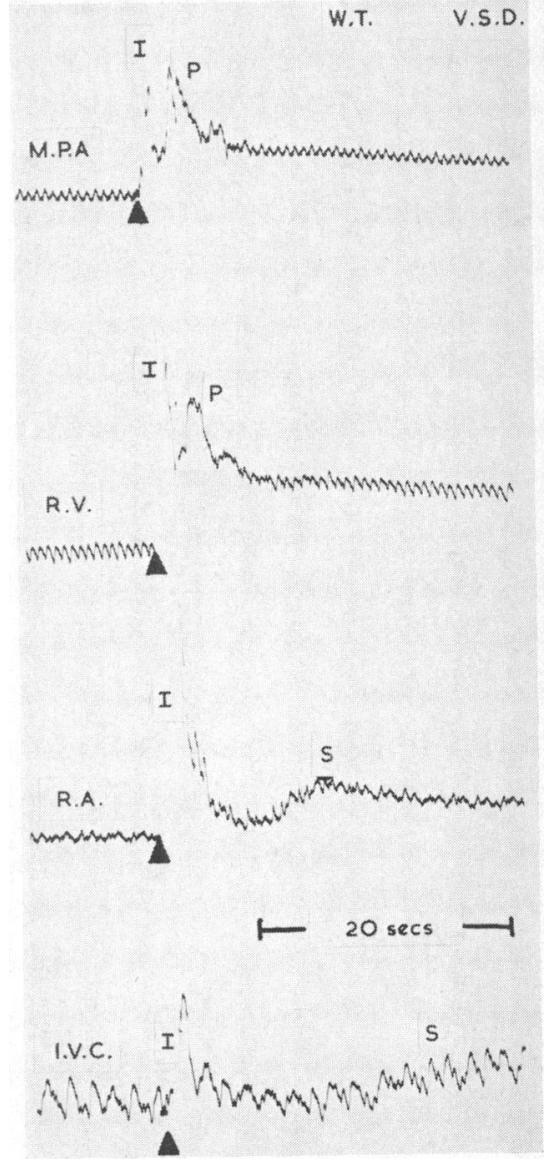

FIG. 2.-Ascorbate dilution curves in ventricular septal defect. Catheter-tip electrode and injection in inferior vena cava (IVC), right atrium (RA), right ventricle $(\mathrm{RV})$, and pulmonary artery (MPA).

$I=$ deflection caused by ascorbic acid leaving the catheter;

$\mathrm{S}=$ systemic recirculation peak;

$\mathbf{P}=$ pulmonary recirculation peak, present in the chambers that receive blood shunted from the left. 
FIG. 3.-Patterns of ascorbate dilution curves recorded in atrial septaldefect (ASD), ventricular septal defect (VSD), and patent ductus arteriosus (PDA), when the catheter-tip electrode is in the right atrium (RA), right ventricle (RV), and pulmonary artery (PA). The pulmonary recirculation peak $(P)$ occurs in the chambers that receive blood shunted from the left.

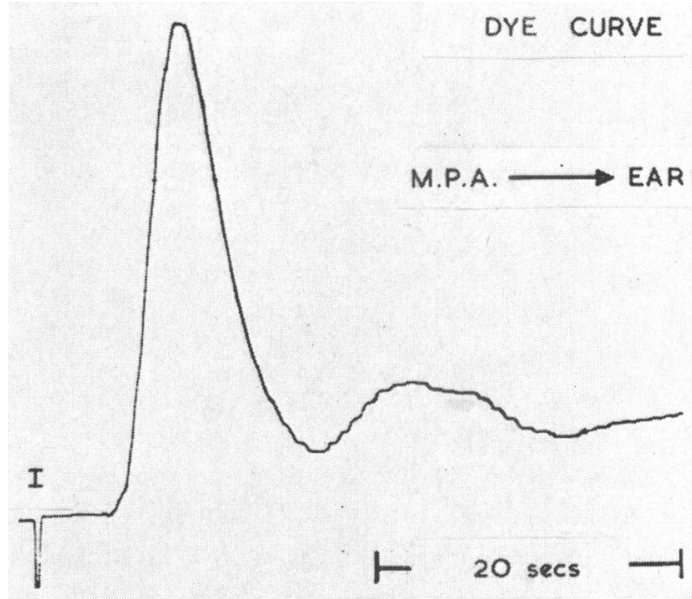

FIG. 4.-Ear-piece blue dye curve in ventricular septal defect with small shunt. 


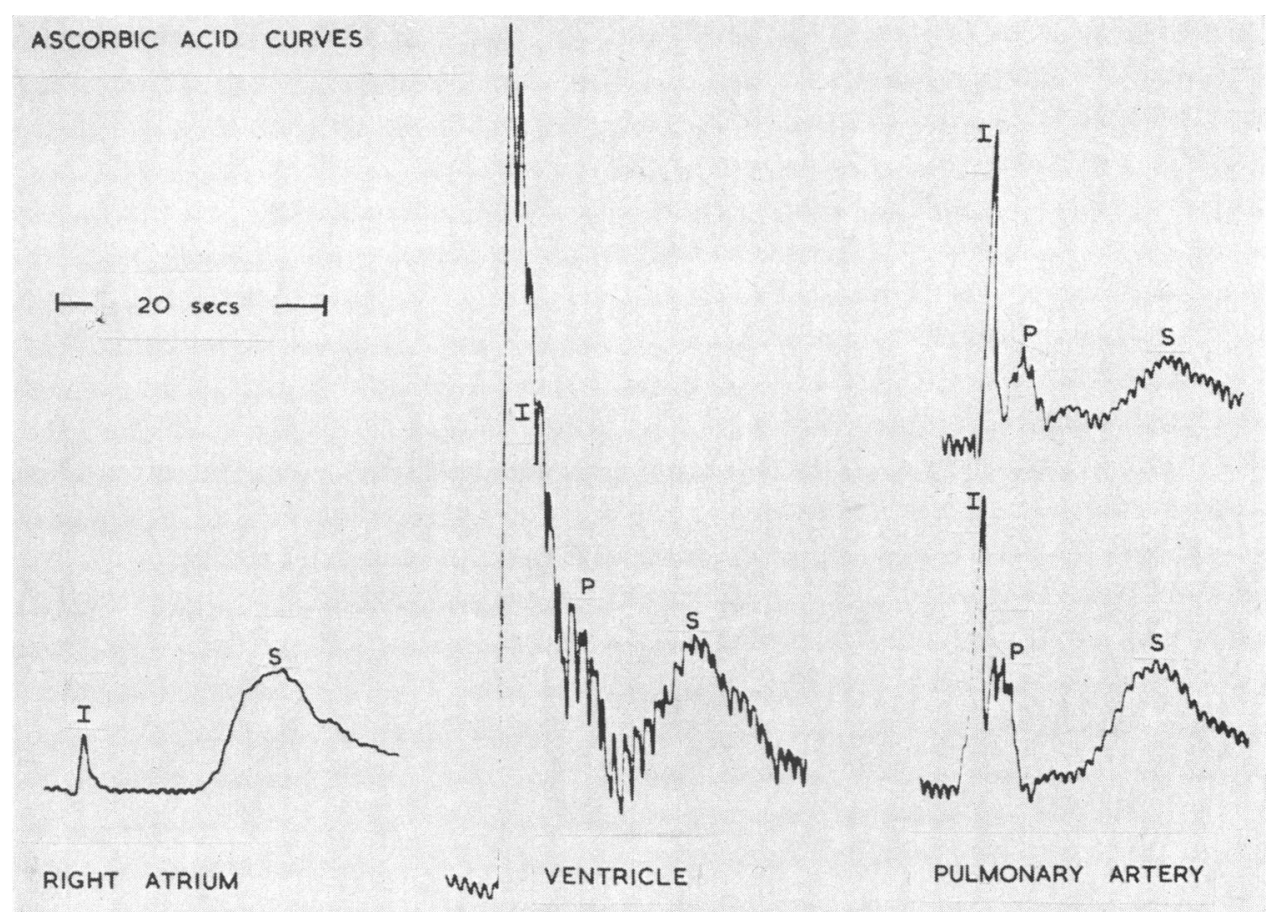

Fig. 5.-Ascorbate dilution curves in ventricular septal defect with small shunt. The pulmonary recirculation peak $(P)$ is present in the right ventricular and pulmonary arterial curves.

Arterial Ascorbate Dilution Curves. Brachial arterial ascorbate and ear-piece (Gabe and Shillingford, 1961) dye dilution curves may be recorded simultaneously after the injection of a mixture of ascorbic acid and Coomassie blue. When the tracings are compared it is evident that the ratio of recirculation peak height to primary circulation peak height is often smaller in the ascorbate than in the dye curve. This may be caused by the more rapid absorption of ascorbate ions during the primary circulation. In most respects the curves are alike, and they may be interchanged for qualitative diagnostic purposes (Fig. 7).

Flow Pulses. When the baseline pulsation from the brachial artery was first recorded it was seen to resemble the pressure pulse (Fig. 9). This suggested that it might be caused by some related factor such as the velocity of blood flow over the electrode or fluctuation in the distension of the artery.

The response to occlusion of the brachial artery, at first above and then below the site of the electrode, was observed (Fig. 10). Each manœuvre would abolish blood flow over the electrode, but only the proximal occlusion would remove the effects of pressure and fluctuating distension. The pulsations disappeared in the same manner, suggesting that the cause was blood flow over the electrode. It is generally known that the velocity of fluid over an amperometric electrode affects the current, and in 1947 Müller suggested that in his apparatus the change in current was proportional to the logarithm of the flow velocity.

Experimental work yet to be published (Hepburn, 1963) confirms that the baseline pulsation does in fact record changes in the velocity of flow over the electrode. In vitro, the relation between the velocity and the current change is non-linear, but for flow pulsations less than three-quarters of maximum flow the relation approaches linearity. In the brachial and pulmonary arteries of man it is possible that the blood-flow changes are slow enough to be recorded without serious time 

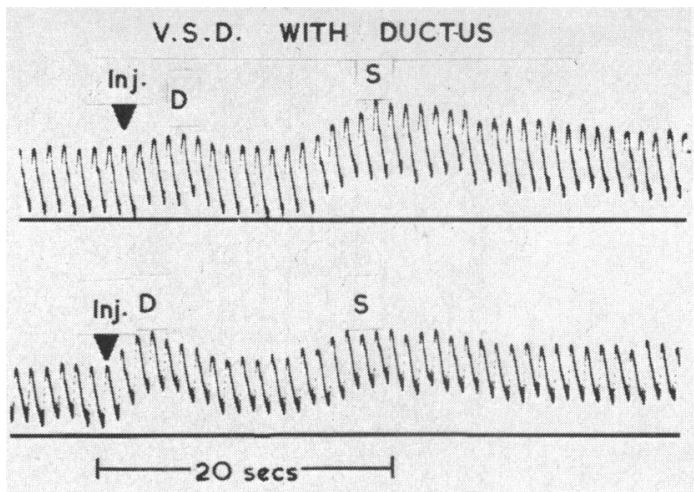

Fig. 6.-Ventricular septal defect with patent ductus arteriosus. Ascorbate dilution curves. Ascorbic acid injected into the aorta. Electrode in the main pulmonary artery. Upper tracing: Injection (Inj) close to the aortic valve. Some ascorbic acid flows through the ductus and causes the deflection (D). The remaining ascorbate passes around the systemic circulation and causes the deflection (S). Lower tracing: Injection close to the orifice of the ductus. The ductus-flow deflection (D) has increased in relation to the systemic-flow deflection (S).
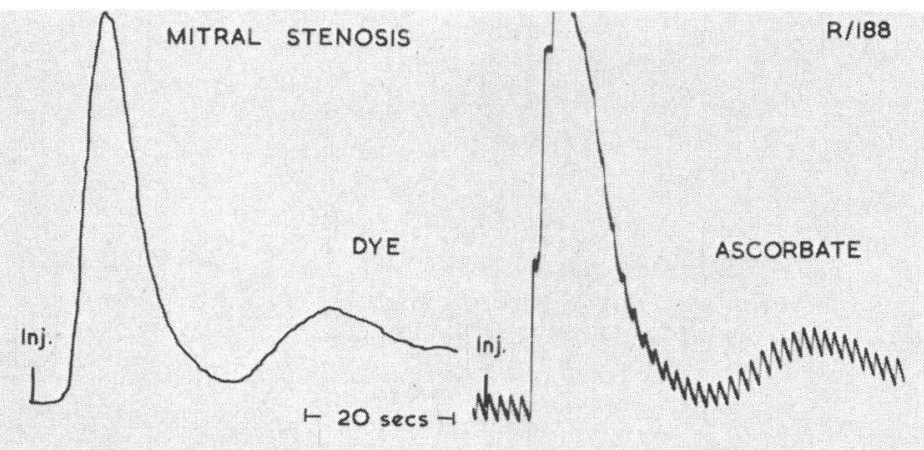

MITRAL INCOMPETENCE

$R / 183$
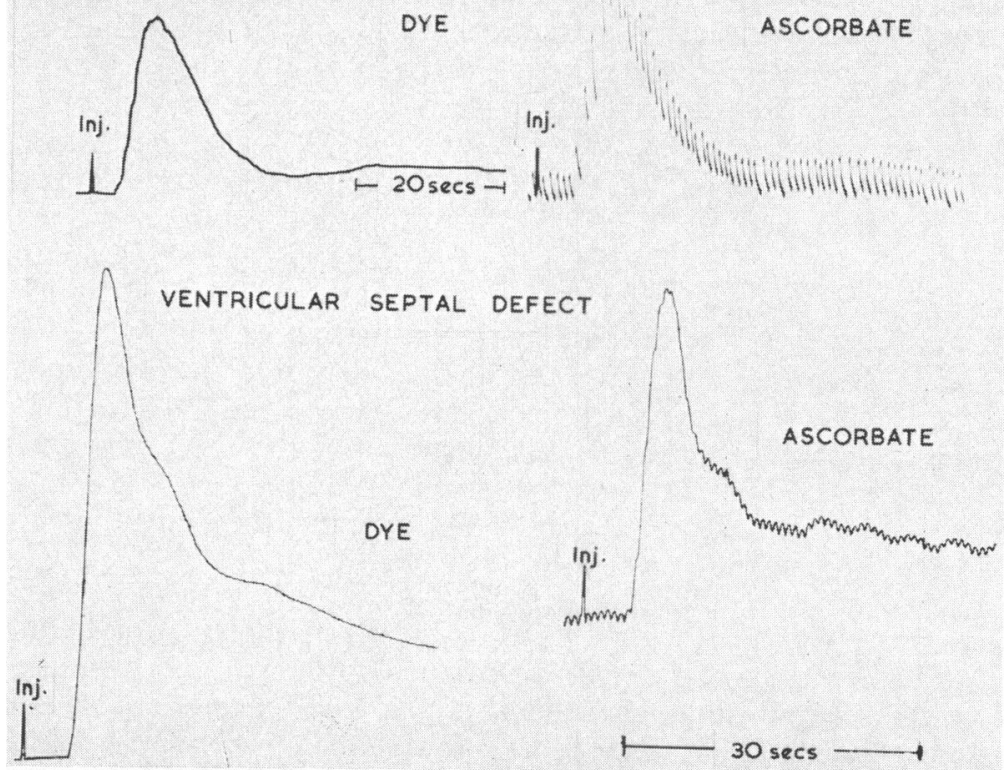

FIG. 7.-Simultaneously recorded ear-piece blue-dye and brachial arterial ascorbate dilution curves in mitral stenosis, mitral regurgitation, and ventricular septal defect. 
distortion. If this is the case, the flow-pulse has a useful place in clinical practice, even if calibration should prove impossible.

\section{CASE RePorts}

Two patients with rheumatic heart disease were investigated by right- and left-heart catheterization. The first (R179, Fig. 11), had an unsuccessful mitral valvotomy nine years before and was extremely disabled by dyspnœa resulting from pulmonary hæmosiderosis and mitral and aortic valvular disease. The pulmonary vascular resistance measured 6 units. The second patient (R189, Fig. 12) had mitral regurgitation but the symptoms were of recent onset and were not severe enough to make her wish to rest after climbing three flights of stairs. The pulmonary vascular resistance measured 5 units. The simultaneously recorded pulmonary arterial pressure and flow pulses from the two patients are shown in Fig. 11 and 12. It may be seen that, while the levels of pressure are not greatly different, the time relations between the pressure and flow pulses are very different in the two cases. In the patient with hæmosiderosis the maxima and minima of pressure and flow occur close together in time, and in the patient with few symptoms they are out of phase. It is possible that the pressure/flow relation found in the patient with hæmosiderosis marked her as having a much higher pulmonary resistance to pulsatile blood flow than the patient with few symptoms, even though there was little difference in their pulmonary vascular resistances estimated from the formula for averaged values:

Mean pulmonary arterial pressure $(\mathrm{mm} . \mathrm{Hg})-$ Mean left atrial pressure $(\mathrm{mm} . \mathrm{Hg})$

Mean cardiac output (1./min.)

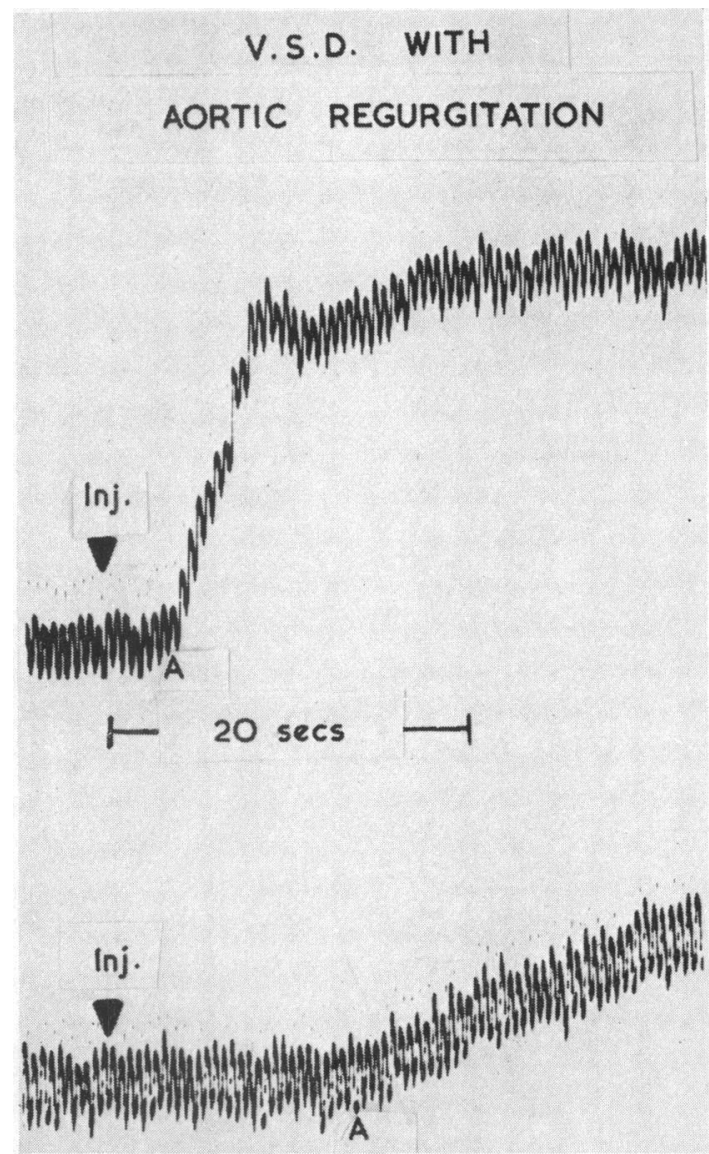

FIG. 8.-Ventricular septal defect with aortic regurgitation. Ascorbate dilution curves. Ascorbic acid injected into the aorta. Electrode in the main pulmonary artery. Upper tracing: Injection (Inj) close to the aortic valve. $A=$ arrival of ascorbic acid at the electrode. Appearance time $=4$ seconds. Lower tracing: Injection (Inj) close to the site of the closed ductus. Appearance time $=16$ seconds.

\section{Discussion}

The detection and localization of left-to-right shunts at cardiac catheterization is simplified by the ease with which it is now possible to inject indicator through a cardiac catheter and to record the dilution curve at the tip of the catheter. It is easier and quicker to practise this method than it is to use cuvette-sampling systems and two-catheter techniques, or angiocardiography. For qualitative indicator-dilution work the amperometric method has certain advantages over the photoelectric methods. The injection of ascorbic acid can be repeated many times without harmful effects, and without precluding oximetry. Cyanosis does not affect the technique. The apparatus is inexpensive, since certain commercial electrocardiographs may be used without modification, and it can be operated by unskilled technicians. The curves are recorded within the circulation, with no loss of blood from sampling, and with no need for catheter-cuvette sampling systems.

The flow-pulse obtained with the intravascular electrode and the amperometric apparatus is worth close study, since it records blood flow accurately enough for the time relation between 

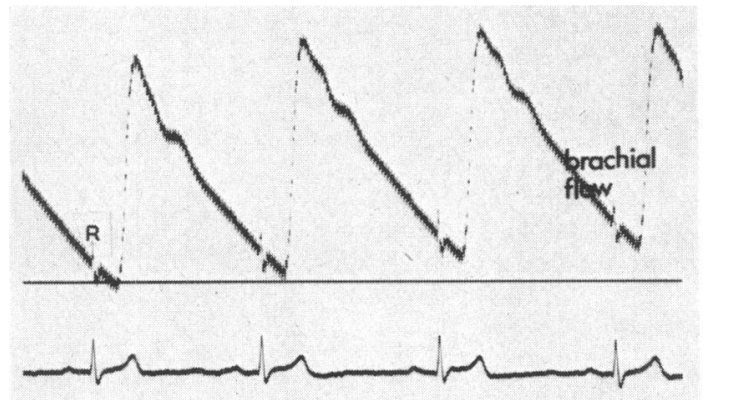

Fig. 9.-Brachial arterial flow and pressure pulses recorded consecutively at cardiac catheterization. $\mathbf{R}=$ the $\mathbf{R}$ wave of electrocardiogram.
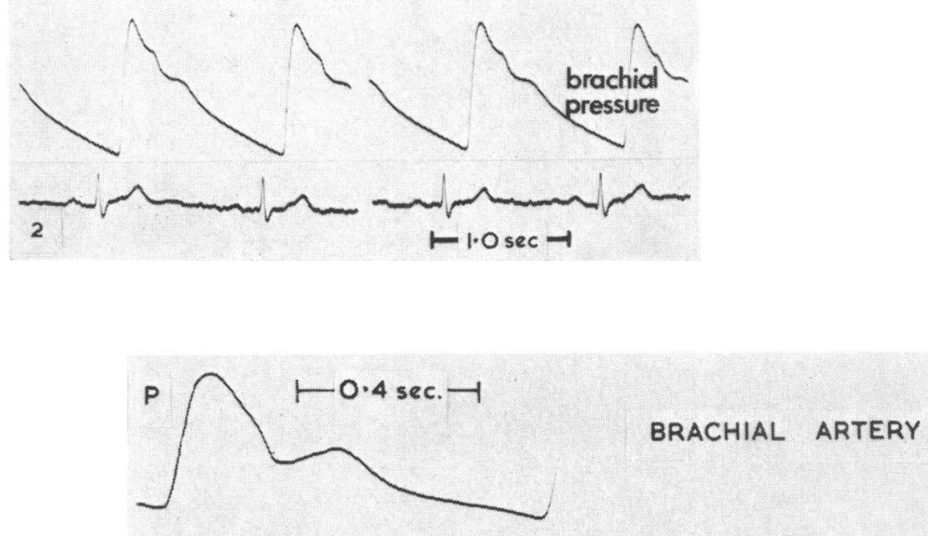

pressure and flow to be examined at cardiac catheterization. This is valuable because it enables the clinician to think in terms of the components of the impedance to pulsatile blood flow instead of being restricted to the resistance concept that is appropriate to steady-state viscous flow.

\section{SUMMARY}

Ascorbic acid, a platinum electrode, and inexpensive amperometric apparatus may be used to demonstrate small left-to-right shunts, to diagnose aorto-pulmonary communications, and to 

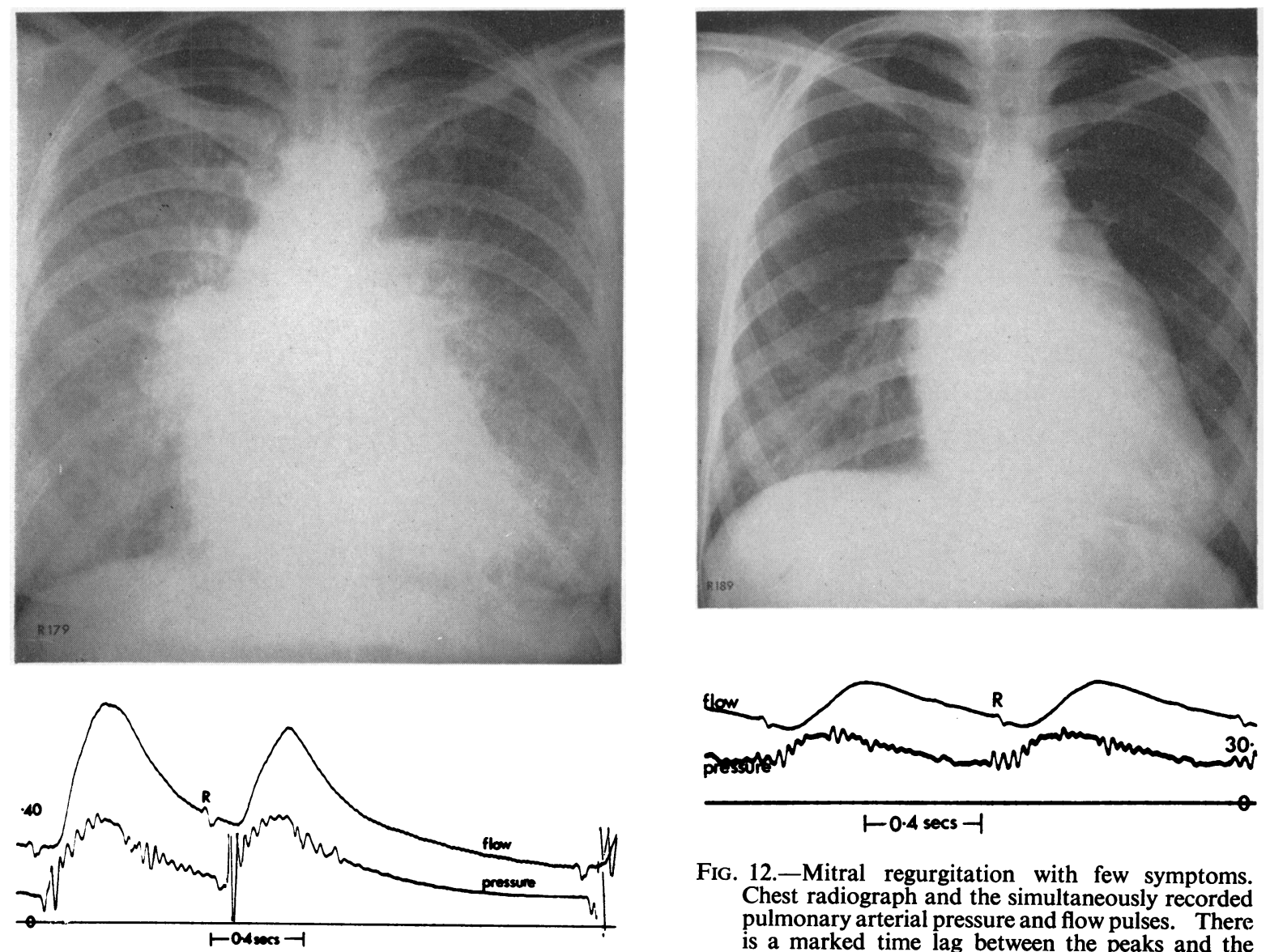

FIG. 12.-Mitral regurgitation with few symptoms. Chest radiograph and the simultaneously recorded pulmonary arterial pressure and flow pulses. There is a marked time lag between the peaks and the troughs of the two pulses $(R=$ electrocardiogram

FIG. 11.-Severe chronic rheumatic heart disease with pulmonary hæmosiderosis. Chest radiograph and the simultaneously recorded pulmonary arterial pressure and flow pulses. The peaks and the troughs of the two pulses occur close together in time $(R=$ electrocardiogram $\mathbf{R}$ wave).

record arterial dilution curves. For qualitative purposes the method has advantage over photoelectric catheter-sampling techniques. The intravascular electrode and the amperometric apparatus may be used to record patterns of arterial blood flow in man. The flow-pulse is recorded accurately enough for the time relation between pressure and flow to be examined at cardiac catheterization.

The authors wish to thank Dr. A. G. Morrow and Dr. P. L. Frommer, of the National Heart Institute, Bethesda, Md., U.S.A., for their unfailing help and kindness, and Professor R. E. Tunbridge and Mr. G. H. Wooler for making this study possible. The Nuffield Foundation, the Medical Research Council, the Endowment Fund of the General Infirmary at Leeds, and private benefactors have generously provided funds for salaries and apparatus.

\section{REFERENCES}

Clark, L. C., Jr. (1960). Trans. Amer. Soc. artif. intern Org., 6, 348.

Trans. Amer. Soc. artif. intern
, and Bargeron, L. M., Jr. (1959). Surgery, 46, 797.

Frommer, P. L., Pfaff, W. W., and Braunwald, E. (1961). Circulation, 24, 1227.

Gabe, I., and Shillingford, J. (1961). Brit. Heart J., 23, 271.

Hay, G. A., Hepburn, F., and Nixon, P. G. F. (1963). In preparation.

Hepburn, F. (1963). In preparation.

Müller, O. H. (1947). J. Amer. chem. Soc., 69, 2992.

Nixon, P. G. F., and Snow, H. M. (1960). Lancet, $2,582$.

Pfaff, W. W., Frommer, P., and Morrow, A. G. (1960). Surg. Forum, 11, 147. 